\title{
Vortex state and effect of anisotropy in sub-100-nm magnetic nanodots
}

\author{
J. Mejía-López \\ Facultad de Física, Pontificia Universidad Católica de Chile, Casilla 306, Santiago 6904411, Chile \\ D. Altbir \\ Departamento de Física, Universidad de Santiago de Chile, USACH, Avenida Ecuador, \\ 3493 Santiago, Chile \\ A. H. Romero \\ CINVESTAV, Unidad Querétaro, Libramiento Norponiente No. 2000 Real de Juriquilla, \\ 76230 Querétaro, Qro., México \\ X. Batlle \\ Departament de Física Fonamental, Universitat Barcelona, Avenida Diagonal 647, 08028 Barcelona, \\ Catalonia, Spain
}

Igor V. Roshchin, Chang-Peng Li, and Ivan K. Schuller

Physics Department, University of California, San Diego, La Jolla, California 92093-0319

(Received 5 June 2006; accepted 17 August 2006; published online 29 November 2006)

\begin{abstract}
Magnetic properties of Fe nanodots are simulated using a scaling technique and Monte Carlo method, in good agreement with experimental results. For the 20 -nm-thick dots with diameters larger than $60 \mathrm{~nm}$, the magnetization reversal via vortex state is observed. The role of magnetic interaction between dots in arrays in the reversal process is studied as a function of nanometric center-to-center distance. When this distance is more than twice the dot diameter, the interaction can be neglected and the magnetic properties of the entire array are determined by the magnetic configuration of the individual dots. The effect of crystalline anisotropy on the vortex state is investigated. For arrays of noninteracting dots, the anisotropy strongly affects the vortex nucleation field and coercivity, and only slightly affects the vortex annihilation field. (C) 2006 American Institute of Physics. [DOI: 10.1063/1.2364599]
\end{abstract}

\section{INTRODUCTION}

Recently, significant attention has been focused on the study of lithographically produced magnetic nanostructures. ${ }^{1}$ This is driven by interest in the properties of nanoscaled materials. This is particularly interesting in magnetism because relevant magnetic length scales are nanometric and magnetic energies for nanoscaled magnets are close to thermal energies. In addition, nanometer-sized structures have potential applications in nonvolatile magnetic memory devices or high-resolution magnetic field sensors. ${ }^{2}$ Arrays of discrete patterned magnetic elements, such as magnetic pillars, rings, and dots, have been proposed as a new generation of ultrahigh density patterned magnetic storage media. ${ }^{3}$ These nanostructures exhibit a variety of magnetic configurations: leaf or flower, in which the magnetization reaches high values close to saturation, and low magnetization configurations in which in-plane curling spin configuration together with an out-of-plane magnetization form a so-called vortex. ${ }^{4}$ For cylindrical dots, there is a critical diameter that depends on the height of the dot and the intrinsic properties of the material. When the diameter $D$ is larger than this critical size but smaller than a domain wall width, the magnetic moment configuration changes from a single domain to a vortex as the in-plane magnetic field is reduced from saturation. The vortex nucleates at one edge of the dot, moves across it, and annihilates on the other side. ${ }^{5}$ In these systems, when the center-to-center distance $d$ between the dots is large enough, i.e., $d \gtrsim 2 D$, the interaction between them becomes negligible. ${ }^{6,7}$ In this case, the magnetic structure within each dot is determined mostly by the internal interactions: the direct exchange between nearest neighbors, the classical dipolar coupling, the crystalline anisotropy, and the Zeeman energy due to an applied magnetic field.

A microscopic theoretical determination of the magnetic configuration of $10-100 \mathrm{~nm}$ structures is out of reach of present standard computational facilities, because of the large number of magnetic moments within such nanostructures. This problem can be solved by using a scaling technique recently developed, ${ }^{8}$ which was applied to a calculation of the phase diagram of magnetic configurations of cylindrical dots of height $H$ and diameter $D$. Smaller structures were used with the exchange interaction scaled down by a factor $x<1$, i.e., $J^{\prime}=J x$, and the dot diameter and height given by $D^{\prime}=D x^{\eta}$ and $H^{\prime}=H x^{\eta}$, respectively, with $\eta \approx 0.55$.

In this paper, we present studies of the magnetic configuration of cylindrical nanodots. After testing the applicability of the scaling technique to this system, we use it to simulate the magnetic configuration of cylindrical nanodots in an inplane magnetic field. ${ }^{9,10}$ The interactions between the dots as a function of separation is studied to determine the geometry for which the dots have negligible interaction. For such noninteracting dots, we investigate the effect of anisotropy on the hysteresis. We compare results of these simulations with 
superconducting quantum interference device (SQUID) magnetometry of hexagonal arrays of sub-100-nm Fe dots covering $\sim 1 \mathrm{~cm}^{2}$. 11,12

\section{MODEL}

The system studied consists of cylindrical magnetic dots, with diameter $D$ and height $H$. While a variety of dot sizes are simulated, in this paper we present only the results for $\mathrm{Fe}$ dots with $D=65 \mathrm{~nm}$ and $H=20 \mathrm{~nm}$, corresponding to one of the samples studied experimentally.

The internal energy $E_{\text {tot }}$ of a single dot with $N$ magnetic moments can be written as

$$
E_{\mathrm{tot}}=\frac{1}{2} \sum_{i \neq j}\left(E_{i j}-J_{i j} \hat{\boldsymbol{\mu}}_{i} \cdot \hat{\boldsymbol{\mu}}_{j}\right)+E_{H},
$$

where the dipolar energy is

$$
E_{i j}=\frac{\boldsymbol{\mu}_{i} \cdot \boldsymbol{\mu}_{j}-3\left(\boldsymbol{\mu}_{i} \cdot \hat{\mathbf{n}}_{i j}\right)\left(\boldsymbol{\mu}_{j} \cdot \hat{\mathbf{n}}_{i j}\right)}{r_{i j}^{3}},
$$

and $r_{i j}$ is the distance between $\boldsymbol{\mu}_{i}$ and $\boldsymbol{\mu}_{j}$, and $\hat{\mathbf{n}}_{i j}$ is the unit vector along the direction that connects the two magnetic moments. $J_{i j}$ is the exchange coupling, which is assumed to be nonzero only for nearest neighbors. $\hat{\boldsymbol{\mu}}_{i}$ is a unit vector along the direction of $\boldsymbol{\mu}_{i} \cdot E_{H}=-\sum_{i=1}^{N} \boldsymbol{\mu}_{i} \cdot H$ is the Zeeman energy. So far the magnetic crystalline anisotropy is ignored and included in the Sec. III C, where each type of anisotropy is specified explicitly and its effect studied in detail.

This technique combined with our scaling method, when applied to granular Fe, using $\left|\boldsymbol{\mu}_{i}\right|=\mu=2.2 \mu_{B}$, a lattice parameter $a_{0}=2.8 \AA$ and $J=42 \mathrm{meV}$ (Ref. 13), gives a Curie temperature for bulk Fe $(1043 \mathrm{~K})$ in good agreement with experiment.

For dot sizes considered in this paper, $N$ would be larger than $10^{6}$. So, we replace the dot with a smaller one according to the scaling technique described above with $\eta=0.57$, as used in Refs. 9, 10, and 14. Correspondingly, we also scale the exchange interaction by a factor $x \equiv J^{\prime} / J=2.1 \times 10^{-3}$, i.e., we replace $J$ with $J^{\prime}=0.09 \mathrm{meV}$ in the expression for the total energy. This approach is tested using different values of the scaling parameter $x$ and shown to be independent of it. For each set of simulations the value of $x$ is chosen so as to obtain meaningful results in a reasonable computational time.

In this work, we present hysteresis curves obtained using this scaling technique and analyze the in-plane magnetization as a function of applied in-plane magnetic field. It is important to realize that, due to the nonequilibrium situation when measuring hysteresis loops for a magnetic system, the number of Monte Carlo steps used is a critical issue. We follow the procedure used by many other authors ${ }^{15,16}$ in which the number of Monte Carlo steps are changed until a fair agreement with the experimental results is obtained. Then, the number of Monte Carlo steps is kept fixed and all other variables are changed. Monte Carlo simulations are carried out using the Metropolis algorithm with local dynamics and single-spin flip methods. ${ }^{16}$ The new orientation of the magnetic moment is chosen arbitrarily with a probability $p=\min \left[1, \exp \left(-\Delta E / k_{B} T\right)\right]$, where $\Delta E$ is the change in energy

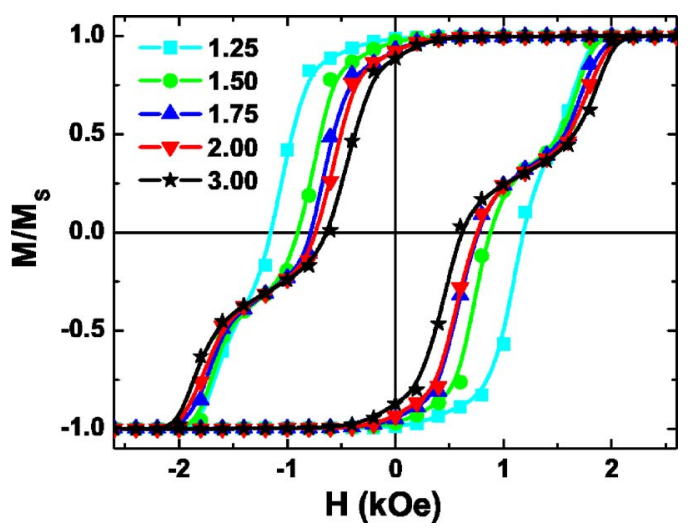

FIG. 1. (Color online) Hysteresis loops of two interacting magnetic dots with $D=65 \mathrm{~nm}$ and $H=20 \mathrm{~nm}$. Squares illustrate the results for two dots with a center-to-center distance $d=1.25 D$, circles corresponds to $d=1.5 D$, up triangles correspond to $d=1.75 D$, down triangles correspond to $d=2 D$, and stars to $d=3 D$, which coincides with the case of noninteracting dots. Lines are guide to the eye.

due to the reorientation of the spin, $k_{B}$ is the Boltzmann constant, and $T$ is the temperature. An interesting point to consider is the temperature at which our simulations are carried out. Because of the size scaling we must scale down the temperature also. Mean field theories imply that the Curie temperature of a system is proportional to $J$; therefore the temperature should be scaled linearly with $J$. Therefore, in our calculations we use $T^{\prime}=0.021$, which represents a "real" temperature $T=T^{\prime} / x=10 \mathrm{~K}$, at which the experimental hysteresis loops are measured.

In the simulation, the magnetization curve is started at $H=2.6 \mathrm{kOe}$ applied along the [100] crystallographic direction, labeled the $X$ axis, with an initial configuration in which most of the magnetic moments point along this direction. We define $M_{s}$ as the magnetization at the maximum applied field (2.6 kOe), $M_{r}$ the remanent magnetization, and $H_{c}$ the coercivity. Field steps of $\Delta H=0.2$ kOe are used in all calculations. Typically, we perform $3.4 \times 10^{5}$ Monte Carlo steps per spin for a complete hysteresis loop, and 5-20 different seeds for the random number generator, depending on the needs of each calculation. The fields at which the vortex core appears inside and leaves the dot are called the nucleation $\left(H_{n}\right)$ and annihilation $\left(H_{a}\right)$ fields, respectively.

\section{RESULTS AND DISCUSSION}

\section{A. Interdot distance and interaction}

The first issue we address is the effect of interactions with neighboring dots on the magnetic properties of a particular dot. Earlier this was considered for much larger dot sizes (dot diameter and height, interdot distance). ${ }^{7,17-22}$ Since the interdot interaction depends on all three sizes, ${ }^{18}$ and because these sizes for our sub-100-nm dots approach characteristic magnetic length scales - domain wall width and exchange length, it is important to investigate the effect of interdot interaction at this scale.

We simulate the response of two dots to a magnetic field applied along the axis connecting the dot centers, as a function of the center-to-center distance between them, $d$, using the scaled size of $D^{\prime}=1.95 \mathrm{~nm}$ and $H^{\prime}=0.6 \mathrm{~nm}$. Figure 1 


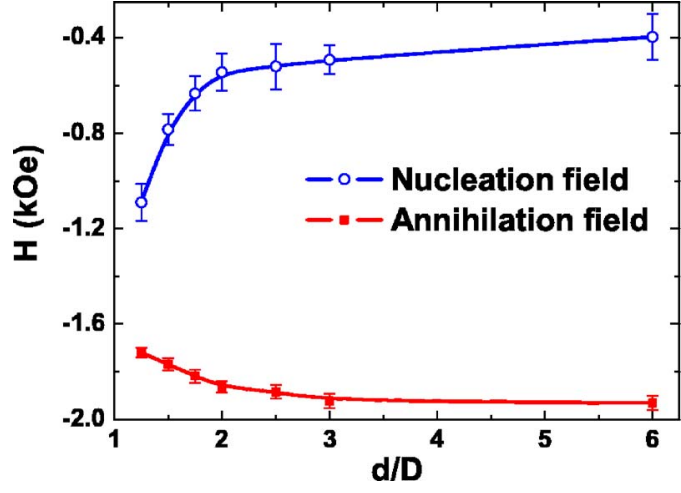

FIG. 2. (Color online) Vortex nucleation $(\bigcirc)$ and annihilation ( $\square$ ) fields calculated for two dots as a function of $d / D$. Lines are guide to the eye.

shows the average of the results obtained for 20 different random initial configurations. The narrowing of the hysteresis curve in the middle is due to the presence of a vortex state.

Figures 2 and 3 illustrate how the vortex nucleation, annihilation, and coercive (zero magnetization) fields and the remanent magnetization depend on the distance between the dots. The closer the dots are to each other, the stronger the interaction is, and consequently (Fig. 2) the larger the vortex nucleation field is. When the dot magnetization is mostly aligned in one direction, the interdot dipolar interaction favors such a configuration, hence opposing vortex nucleation, creating an additional energy barrier for the transition to the vortex state, and increasing the vortex nucleation field. The annihilation field is also affected by the interdot interaction, but to a lesser degree. This is attributed to a much weaker dipolar interaction between the dots in a vortex state. Probably for the same reason, we do not observe any effect of the vortex chiralities in the individual dots on the vortex characteristic fields.

Figure 3 shows that the normalized remanence (a) and coercivity (b) decrease when $d$ increases for this two-dot system, leveling off for distances larger than $3 D$ to the values obtained for a single dot. Error bars are calculated from 20 different realizations of the hysteresis loops. This figure indicates that the further apart the two dots are, the more simi-
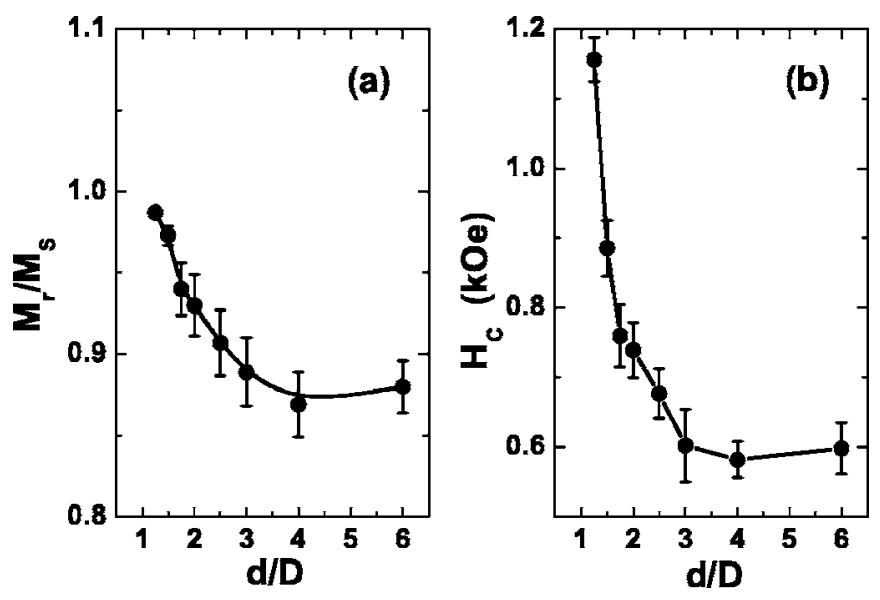

FIG. 3. Normalized remanence (a) and coercivity (b) of two interacting dots as a function of $d / D$. Lines are guide to the eye.

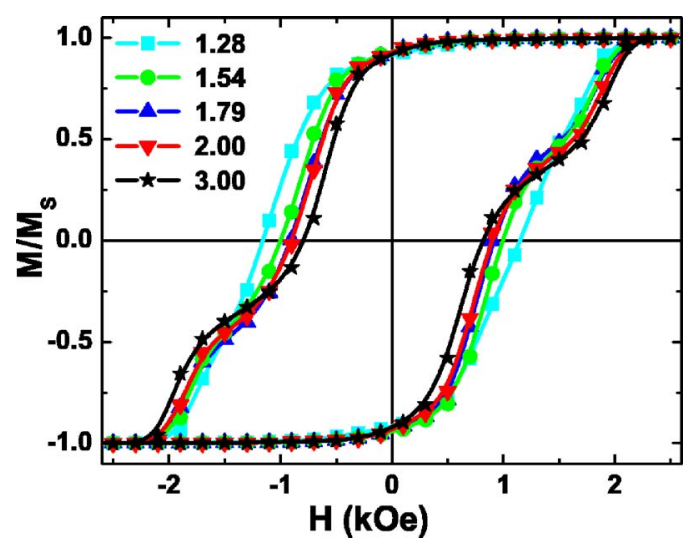

FIG. 4. (Color online) Hysteresis loops of the central dot interacting with its six nearest neighbors in an hexagonal dot array for different center-to-center distances. The curve for $d=3 D$ is identical to the one for the noninteracting dots. Lines are guide to the eye.

lar the dots properties are to those of a single dot. When $d$ $<2 D$, the interaction between two magnetic dots is important and can significantly modify magnetic reversal. While it is only for $d \geqslant 3 D$ that the hysteresis loop coincides well with the one for a single noninteracting dot, for $2 D \leqslant d$ $<3 D$, the shape of the hysteresis loop is very similar to the noninteracting case. The coercivity and the remanent magnetization vary less than $8 \%$ if the center-to-center distance changes from $2 D$ to $3 D$. Thus, for $d \geqslant 2 D$ the magnetic properties of two interacting dots are well described by a statistical average of the magnetic properties of noninteracting dots.

The results obtained for hexagonal dot arrays are very similar to those obtained for just two dots. The hysteresis loops of the central dot $(D=65 \mathrm{~nm}, H=20 \mathrm{~nm})$ of 7 dots in a hexagonal lattice as a function of the center-to-center distance are presented in Fig. 4.

The higher-order neighbors play even smaller role: The center-to-center distances to the second and the third neighbors are $\sqrt{3} d$ and $2 d$, respectively. For $d=2 D$, these are $2 \sqrt{3} D$ and $4 D$. As can be seen in Figs. 2 and 3, the effect of interaction at these distances is negligible, despite the larger number of higher order neighbors. It is noteworthy that the edge-to-edge distances in the case of $d=2 D$ are equal to $D$, $\sim 2.4 D$, and $3 D$ for the first, second, and third nearest neighbors, respectively, which helps us understand the small effect of higher order neighbors.

For the rest of the discussion we assume a system with $d \gtrsim 2 D$, thus, a noninteracting system. This is a reasonable assumption for the experimentally studied samples with $d$ $\approx 2 D$.

\section{B. Comparison with experimental results}

We compare results of these simulations with SQUID magnetometry of hexagonal arrays of sub-100-nm Fe dots on $\mathrm{Si}$ substrates. These arrays are fabricated using selfassembled nanopores in anodized alumina as a mask. ${ }^{11}$ The in-plane magnetization of the dots is measured ${ }^{12}$ at $T$ $=10 \mathrm{~K}$ using a SQUID magnetometer. The experimental system presented in this work consists of a hexagonal array of 


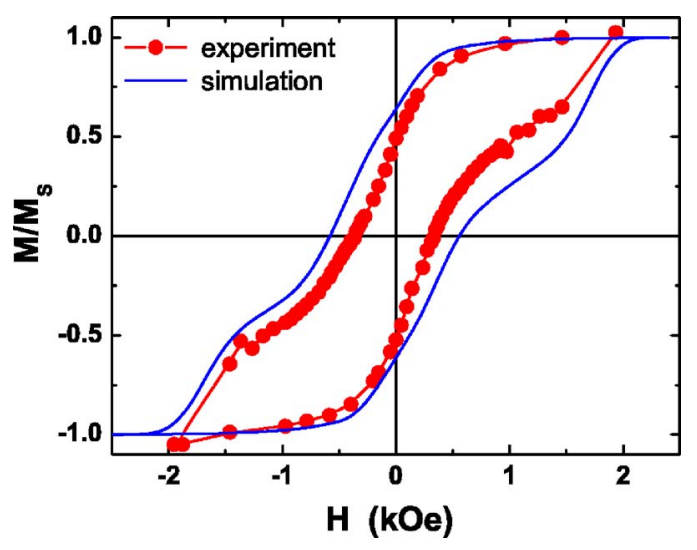

FIG. 5. (Color online) Experimental hysteresis curve (dots) for an array of dots with $D=20 \mathrm{~nm}, H=65 \mathrm{~nm}$, and $d=110 \mathrm{~nm}$, and calculated hysteresis curve (continuous line) for the same size noninteracting dot, obtained as an average of curves for dots with $4 \%$ and $5 \%$ surface defects.

Fe dots with the nominal sizes of the simulated dots: $D$ $=65 \mathrm{~nm}, H=20 \mathrm{~nm}$, and $d=110 \mathrm{~nm}$. Since the dots measured in the experiment have a number of defects, we performed calculations with surface defects modeled as a fixed percentage of vacant surface sites, i.e., sites with no spin. Inclusion of surface defects results in a decreased remanent magnetization and vortex nucleation field compared to those of an ideal dot, while producing very little or no effect on the coercivity. We find that the average of the curves obtained for $4 \%$ and $5 \%$ of surface defect distribution provides a good fit to the shape of the hysteresis curve measured in the experiment. We realized 10 different simulations for each defect concentration. Our results and experimental data are illustrated in Fig. 5. The difference between simulated and experimental values of coercive field is attributed to the distribution of dot sizes and imperfections of the shape of the dots in the experimental system. Also, the neglected interdot interactions, while not producing qualitative changes to the overall hysteresis loop shape, may contribute to some small quantitative corrections.

It is noteworthy that in these dots the remanent magnetic state obtained by following the major magnetization loop is not a vortex state. While the vortex state corresponds to the global energy minimum, the system can be stuck in a local energy minimum that corresponds to a nonvortex spin configuration. The range of dot sizes for which the vortex state can coexist with other (metastable) states has been analyzed by Metlov and Guslienko. ${ }^{23}$

\section{Anisotropy}

In principle, the magnetic anisotropy of nanosized magnetic structures can be controlled by the choice of material, growth parameters, and fabrication methods. However, this control is rather limited for the $\mathrm{Fe}$ dot arrays fabricated using self-assembled nanopores in anodized alumina. So, we investigate the effect of various types of anisotropy on the vortex appearance, its motion along the dot, and annihilation. Earlier publications ${ }^{24-26}$ compared only one, uniaxial crystalline

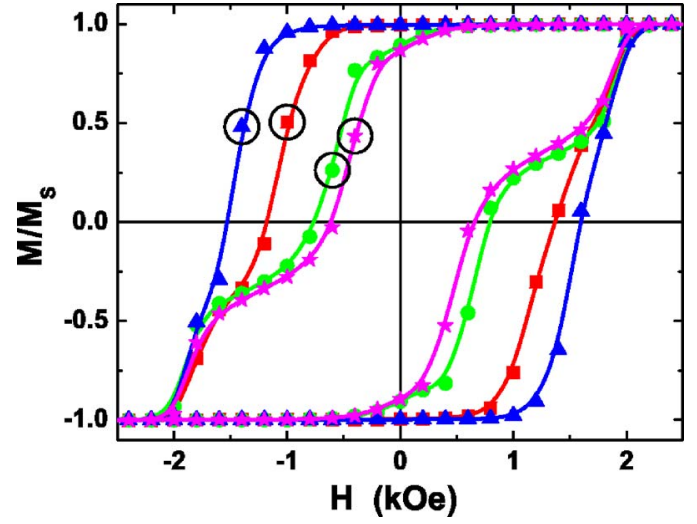

FIG. 6. (Color online) Hysteresis loops of a magnetic dot of $D=65 \mathrm{~nm}$ and $H=20 \mathrm{~nm}$ for four different types of anisotropy: easy-axis (triangles), cubic (squares), easy-plane (circles), and no anisotropy (stars). Points at which the vortex first enters the dot are circled in black.

anisotropy to the case with no anisotropy. We compare hysteresis loops obtained by adding three different types of anisotropy, to the total energy (Eq. (1)), cubic, easy-axis, and easy-plane, with the previously obtained, without anisotropy (stars in Fig. 6).

The cubic crystalline anisotropy (squares in Fig. 6) is given by $E_{K}=K \sum_{i=1}^{N}\left[\alpha_{i}^{2} \beta_{i}^{2}+\beta_{i}^{2} \gamma_{i}^{2}+\gamma_{i}^{2} \alpha_{i}^{2}\right]$, where $\left(\alpha_{i}, \beta_{i}, \gamma_{i}\right)$ are the cosines of the angles that the moment $i, \boldsymbol{\mu}_{i}$, forms with the [100], [010], and [001] axes, respectively. ${ }^{27}$ The easy-plane anisotropy (circles in Fig. 6) is modeled by defining a hard axis along the $Z$ axis, so that the anisotropy energy is given by $E_{K}=K \sum_{i=1}^{N} \cos ^{2} \theta_{i}$, where $\theta_{i}$ is the angle of the magnetic moment $i$ with the $Z$ axis. The uniaxial anisotropy along [100] (triangles in Fig. 6), is modeled by $E_{K}=$ $-K \Sigma_{i=1}^{N} \cos ^{2} \Psi_{i}$, where $\Psi_{i}$ is the angle between the magnetic moment $i$ and the easy axis, $X$. A value of $K=8.44$ $\times 10^{-3} \mathrm{meV}$ per spin, which is close to the Fe bulk value, is used for all anisotropies, with the magnetic field applied along the $X([100])$ axis. The value of the anisotropy constant $K$ is not scaled as explained in Ref. 28.

For each of the anisotropies, the first point at which the vortex core appears inside the dot is circled in black in Fig. 6. In each case, the vortex nucleation field $H_{n}$ is between this point and the previous one. $H_{n}$ is the smallest in the case of a system without anisotropy. Since the easy-plane anisotropy does not break in-plane translational invariance, it enhances the in-plane anisotropy similarly to an enhanced shape anisotropy. For the dot size considered, a quasi-two-dimensional model (i.e., no $Z$-dependent variation of the magnetic moment orientation) favors formation of a single domain state, thus increasing the vortex nucleation field and the coercivity as pointed out by Hoffmann and Steinbauer. ${ }^{29}$

For an in-plane easy-axis anisotropy, the anisotropy energy can be written as the sum of a term that depends on the angle $\phi_{i}$ of the magnetic moment with the plane, and another term that depends on the angle $\psi_{i}$ between the projection of the moment on the plane and the easy axis, 


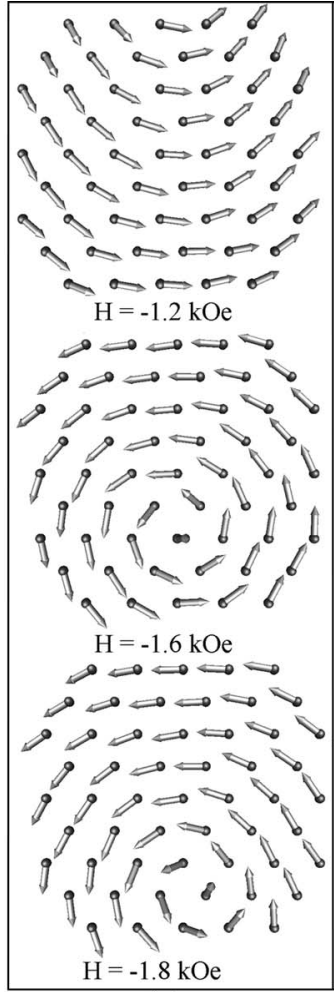

(a)

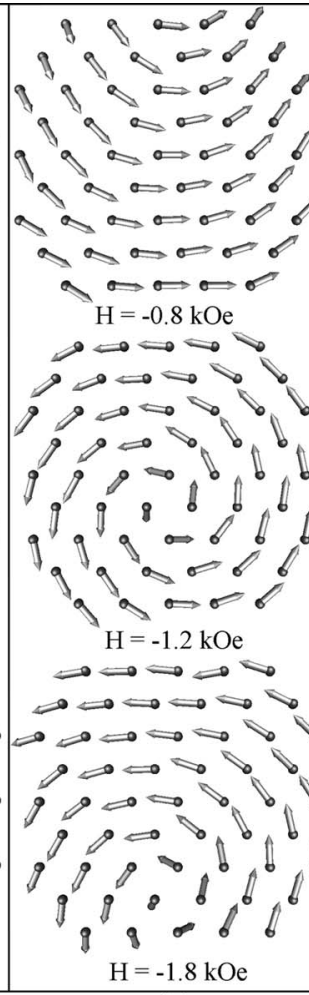

(b)

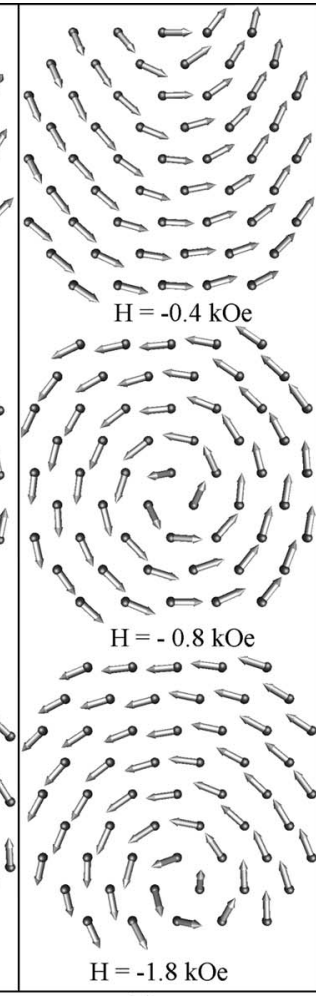

(c)

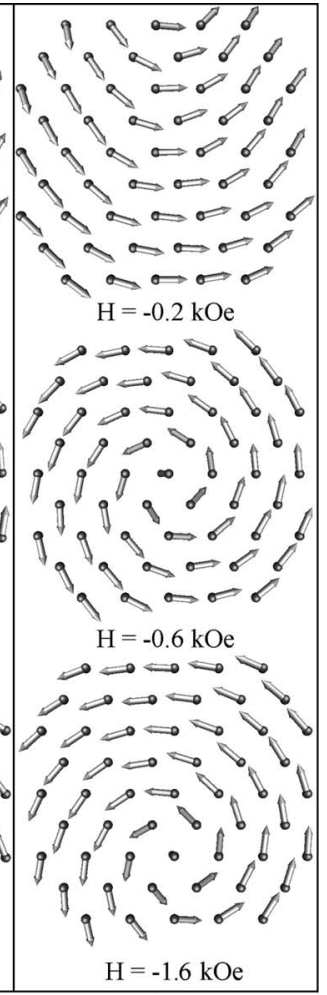

(d)
FIG. 7. Snapshots of the vortex evolution inside the dot with (a) easy-axis, (b) cubic, (c) easy-plane, and (d) without magnetic crystalline anisotropy.

$$
\begin{aligned}
E_{k} & =-K \sum_{i=1}^{N} \cos ^{2} \phi_{i}-K \sum_{i=1}^{N} \cos ^{2} \psi_{i} \\
& =-K \sum_{i=1}^{N}\left(1-\cos ^{2} \theta_{i}\right)-K \sum_{i=1}^{N} \cos ^{2} \psi_{i} \\
& =-N K+K \sum_{i=1}^{N} \cos ^{2} \theta_{i}-K \sum_{i=1}^{N} \cos ^{2} \psi_{i} .
\end{aligned}
$$

Here we use that $\phi_{i}=\pi / 2-\theta_{i}$, and hence, $\cos ^{2} \phi_{i}=\sin ^{2} \theta_{i}$ $=1-\cos ^{2} \theta_{i}$. The first term in Eq. (3) does not depend on the angle. The second term is equivalent to the energy term for the easy-plane anisotropy, hence favoring a single domain state, as described above. The third term favors the spin alignment along the easy axis $X$, which makes the formation of vortex state energetically unfavorable. This explains why the in-plane uniaxial magnetic anisotropy suppresses the vortex state more than the easy-plane anisotropy, thus enhancing the coercivity and increasing the vortex nucleation field.

For each of the three anisotropies and for the case without anisotropy, Fig. 7 shows three spin configurations (from top to bottom): the so-called $C$ state $^{30}$ just before nucleation of the vortex (with spins curling in the shape of the letter $C$ ), the vortex with the core near the center of the dot, and the vortex state just before annihilation. Of these three spin configurations, the anisotropy appears to have little or no effect on the third one, and hence on the vortex annihilation field. The fields at which the first two configurations are realized depends on the anistropy. Hence, the vortex nucleation field and coercivity are different for all four anisotropies. This is in accordance with our earlier discussion. We find here a universal trend: the higher the degree of degeneracy of states that minimize the anisotropy energy term, the more such anisotropy favors the vortex state.

\section{CONCLUSIONS}

In this paper, we present Monte Carlo simulations of magnetic properties of magnetic Fe nanodots. Using an earlier developed scaling technique, we simulate the behavior of dots containing a large number of spins within a relatively short calculational time. We demonstrate that the magnetic properties of a dot array, with center-to-center distances larger than twice the dot diameter and the dots sizes considered in this work $(D=20-120 \mathrm{~nm}, h=20 \mathrm{~nm})$, can be studied by averaging the properties of a single dot calculated from different random seeds. At these distances, the interdot magnetic interactions are relatively weak and can be neglected. Therefore, the magnetic properties of the experimentally studied systems with characteristic sizes similar to those considered here can be treated as properties of a single dot.

Surface defects decrease the vortex nucleation field and the remanent magnetization, but have little or no effect on the coercivity. The magnetic hysteresis curves simulated using a Monte Carlo method with scaling, reproduce well the experimental curves.

The effects of various magnetic anisotropies on vortex state are presented. The anisotropy affects the vortex nucleation field and the coercivity, but has little or no effect on the vortex annihilation field. We establish a universal trend for the effect of anisotropy on the vortex state: the higher the degree of degeneracy of the states that minimize the anisotropy energy, the more such anisotropy favors the vortex state. 


\section{ACKNOWLEDGMENTS}

This research received financial support from FONDECYT under Grant Nos. 1050066, 1050013, and 7050111, and from the Millennium Science Nucleus "Condensed Matter Physics" P02-054F. In Mexico the authors acknowledge the support by CONACYT under Grant No. J-42647-F. In the U.S.A. the support from U.S. AFOSR and the UC MEXUS is acknowledged. One of the authors (A.H.R.) acknowledges the support from MECESUP USA0108, Chile and another author (X.B.) acknowledges the support of the Spanish MEC (PR2003-0149), CYCIT (MAT2003-01124), and the Catalan Dursi (ACI2002-005). Most of the authors calculations were performed on a computational cluster of the Mathematics Department of Universidad de Concepcion. The authors also thank Z.-P. Li for fruitful discussions.

${ }^{1}$ J. I. Martin, J. Nogues, K. Liu, J. L. Vicent, and I. K. Schuller, J. Magn. Magn. Mater. 256, 449 (2003).

${ }^{2}$ J. N. Chapman, P. R. Aitchison, K. J. Kirk, S. McVitie, J. C. S. Kools, and M. F. Gillies, J. Appl. Phys. 83, 5321 (1998).

${ }^{3}$ C. A. Ross et al., J. Vac. Sci. Technol. B 17, 3168 (1999).

${ }^{4}$ T. Shinjo, T. Okuno, K. Shigeto, and T. Ono, Science 289, 930 (2000).

${ }^{5}$ K. Yu. Guslienko and K. L. Metlov, Phys. Rev. B 63, 100403(R) (2001).

${ }^{6}$ C. A. Ross, M. Farhoud, M. Hwang, H. I. Smith, M. Redjdal, and F. B. Humphrey, J. Appl. Phys. 89, 1310 (2001).

${ }^{7}$ M. Grimsditch, Y. Jaccard, and I. K. Schuller, Phys. Rev. B 58, 11539 (1998).

${ }^{8}$ J. d'Albuquerque e Castro, D. Altbir, J. C. Retamal, and P. Vargas, Phys. Rev. Lett. 88, 237202 (2002).

${ }^{9}$ J. Mejía-López, P. Soto, and D. Altbir, Phys. Rev. B 71, 104422 (2005).

${ }^{10}$ D. Altbir, J. d'Albuquerque e Castro, and P. Vargas, Phys. Rev. B 73, 092417 (2006)

${ }^{11}$ For fabrication of magnetic nanodot arrays over macroscopic areas using porous alumina see, e. g., K. Liu, J. Nogues, C. Leighton, I. V. Roshchin, H. Masuda, K. Nishio, and I. K. Schuller, Appl. Phys. Lett. 81, 4434 (2002); C.-P. Li, I. V. Roshchin, X. Batlle, and I. K. Schuller, J. Appl. Phys. 100, 074318 (2006).

${ }^{12}$ The experimental studies of the magnetic nanodot arrays are presented in I. V. Roshchin et al. (unpublished).

${ }^{13}$ C. Kittel, Introduction to Solid State Physics, 6th ed. (Wiley, New York, 1986), pp. 23 and 425.

${ }^{14}$ P. Landeros, J. Escrig, D. Altbir, D. Laroze, J. d'Albuquerque e Castro, and P. Vargas, Phys. Rev. B 71, 094435 (2005).

${ }^{15}$ E. De Biasi, C. A. Ramo, and R. D. Zysler, Phys. Rev. B 65, 144416 (2002).

${ }^{16}$ K. Binder, Rep. Prog. Phys. 60, 487 (1997).

${ }^{17}$ K. Yu. Guslienko, Appl. Phys. Lett. 75, 394 (1999).

${ }^{18}$ K. Yu. Guslienko, S.-B. Choe, and S.-C. Shin, Appl. Phys. Lett. 76, 3609 (2000).

${ }^{19}$ V. Novosad, K. Yu. Guslienko, Y. Otani, H. Shima, and K. Fukamichi, J. Magn. Magn. Mater. 239, 234 (2002).

${ }^{20}$ V. Novosad et al., Phys. Rev. B 65, 060402(R) (2002).

${ }^{21}$ K. Yu. Guslienko, V. Novosad, Y. Otani, H. Shima, and K. Fukamichi, Phys. Rev. B 65, 024414 (2002).

${ }^{22}$ A. J. Bennett and J. M. Xu, Appl. Phys. Lett. 82, 2503 (2003).

${ }^{23}$ K. L. Metlov and K. Yu. Guslienko, J. Magn. Magn. Mater. 242-245, 1015 (2002).

${ }^{24}$ L. D. Buda, I. L. Prejbeanu, U. Ebels, and K. Ounadjela, Comput. Mater. Sci. 24, 181 (2002).

${ }^{25}$ P.-O. Jubert and R. Allenspach, Phys. Rev. B 70, 144402 (2004).

${ }^{26}$ Y. Takagaki and K. H. Ploog, Phys. Rev. B 71, 184439 (2005).

${ }^{27}$ C. Kittel, Introduction to Solid State Physics, 6th ed. (Wiley, New York, 1986), p. 450.

${ }^{28}$ J. Escrig, P. Landeros, J. C. Retamal, D. Altbir, and J. d'Albuquerque e Castro, Appl. Phys. Lett. 82, 3478 (2003).

${ }^{29}$ H. Hoffmann and F. Steinbauer, J. Appl. Phys. 92, 5463 (2002).

${ }^{30}$ In addition to the vortex nucleation mechanism via the $C$ state observed here, other vortex nucleation modes could also be realized in cylindrical dots, depending on their sizes. See, e. g., T. Pokhil, D. Song, and J. Nowak, J. Appl. Phys. 87, 6319 (2000) and Ref. 21. 